\title{
Development, dimensions, reliability and validity of the novel Manchester COPD fatigue scale
}

\author{
K Al-shair, ${ }^{1}$ U Kolsum, ${ }^{1}$ P Berry, ${ }^{2}$ J Smith, ${ }^{1}$ A Caress, ${ }^{3}$ D Singh, ${ }^{1}$ J Vestbo ${ }^{1}$
}

- The pilot and final questionnaires together with additional tables and figures are published online only at http:// thorax.bmj.com/content/vol64/ issue 11

${ }^{1}$ Respiratory Research Group, The University of Manchester, South Manchester University Hospitals NHS Foundation Trust, Manchester, UK; ${ }^{2}$ Global Health Outcomes, GlaxoSmithKline, London, UK; ${ }^{3}$ School of Nursing, Midwifery and Social Work, The University of Manchester,

Manchester, UK

Correspondence to: Dr K Al-shair, Respiratory Research Group, 2nd floor The Education and Research Centre, South Manchester University Hospitals Trust NHS Foundation Trust, Wythenshawe, Manchester M23 9LT, UK Khaled.Al-shair@ postgrad.manchester.ac.uk; alshair02@yahoo.com

Received 16 April 2009 Accepted 2 August 2009

\begin{abstract}
Introduction: Fatigue is a prominent symptom in chronic obstructive pulmonary disease (COPD) and it has distinctive features; however, there is a need for a robust scale to measure fatigue in COPD.

Methods: At baseline, 122 patients with COPD (forced expiratory volume in $1 \mathrm{~s}\left(\mathrm{FEV}_{1}\right) 52 \%$, women $38 \%$, mean age 66 years) completed a pilot fatigue scale covering a pool of 57 items and underwent a range of tests,

including indicators of mood and a short general fatigue questionnaire. All patients responded to the 57 -item scale and it was readministered to a subset of 30 patients. The pilot scale was first subjected to constructive validated shortening steps and then to a principal components analysis.
\end{abstract}

Results: The Manchester COPD fatigue scale (MCFS) consists of 27 items, loading into three dimensions: physical, cognitive and psychosocial fatigue. Internal consistency (Cronbach's $\alpha=0.97$ ) and test-retest repeatability $(r=0.97, p<0.001)$ were tested. It had significant convergent validity, correlating with the FACIT (Functional Assessment of Chronic Illness Therapy) fatigue scale and the fatigue in Borg scale at baseline and after a 6 minute walk distance (6MWD) test $(r=-0.81$, 0.53 and 0.63 , respectively, $p<0.001)$. Its scores were associated with BODE, SGRO (St George's Respiratory Questionnaire) and MRC (Medical Research Council) dyspnoea scores $(r=0.46,0.8$ and 0.51 , respectively, $p<0.001)$. The scale demonstrated meaningful discriminating ability; patients who walked $<350 \mathrm{~m}$ in a $6 \mathrm{MWD}$ test as well as depressed patients $(\geqslant 16$ scores in the Center for Epidemiologic Study on Depression (CES-D) scale) had nearly twice as high fatigue scores as those who walked $\geqslant 350 \mathrm{~m}$ or were not depressed $(p<0.001)$. Conclusion: The MCFS provides a simple, reliable and valid measurement of total and dimensional fatigue in moderate stable COPD.

Fatigue is one of the most prominent and disabling symptoms in chronic obstructive pulmonary disease (COPD) ${ }^{1-3}$ It significantly impairs the ability of patients to function normally ${ }^{34}$ and substantially affects their quality of life. ${ }^{25}$ However, fatigue has been studied to a much lesser extent than, for example, breathlessness, and the lack of a robust scale for this complex symptom in COPD may be one explanation.

Indeed, no questionnaire designed specifically to assess fatigue in patients with COPD exists. The Chronic Respiratory Questionnaire (CRQ) consists of four subscales, one of these for assessing fatigue, ${ }^{1}$ and other general and often simple scales to measure fatigue have been used. ${ }^{6}$ However, these general scales may not adequately capture descriptors of fatigue specific to COPD. Fatigue is a common symptom in other chronic diseases such as rheumatoid arthritis, multiple sclerosis ${ }^{4}$ and asthma. ${ }^{8}$ However, fatigue in any of these diseases has distinctive features. In rheumatoid arthritis fatigue is often associated with pain and in multiple sclerosis with neurological symptoms. ${ }^{4}$ In contrast, fatigue in COPD seems uniquely associated with dyspnoea. ${ }^{4}$ Moreover, patients with COPD have been reported to experience fatigue as a routine feeling, always associated with exertion, while patients with asthma experienced fatigue during exacerbations and it was intensified by exertion. ${ }^{8}$

We have carried out pilot work with a 57-item COPD fatigue scale, which correlated well with validated measures of COPD. Although the questionnaire was easy to use, it seemed to have redundant items and it was time consuming. We therefore aimed to shorten the pilot fatigue scale and to examine the reliability and validity of the final version.

\section{METHODS}

\section{Development of the scale}

The pilot COPD fatigue scale was developed through a collaboration between GlaxoSmithhKline and the clinical research organisation Oxford Outcomes after an extensive review of the published literature, available questionnaires, and following discussion with clinical experts in COPD and 36 patients with COPD. For the development of the initial questionnaire 36 patients (with a defined gender and severity distribution) were selected and took part in three sessions (12 patients in each) with focus group interviews and open discussions on questionnaire items. The scale consisted of a pool of 57 items examining the impact of fatigue in patients with COPD; a copy of the pilot questionnaire can be seen in the online supplement.

\section{Administration of the scale}

In this study, the scale was administered twice, first to the whole group $(n=122)$ and then to a randomly selected subset of 30 of the patients (17 men) after a 4-week interval. On both occasions, patients were clinically stable for at least 4 weeks. A summary of the administration and shortening steps is provided in fig 1 .

\section{Patients}

Patients were recruited from media advertising and the outpatient chest clinic at the North West Lung Centre, South Manchester University Hospital Trust, Manchester. COPD was diagnosed according to GOLD (Global Initiative for Chronic 
Figure 1 Flow chart illustrating the development of the Manchester chronic obstructive pulmonary disease (COPD) fatigue scale.

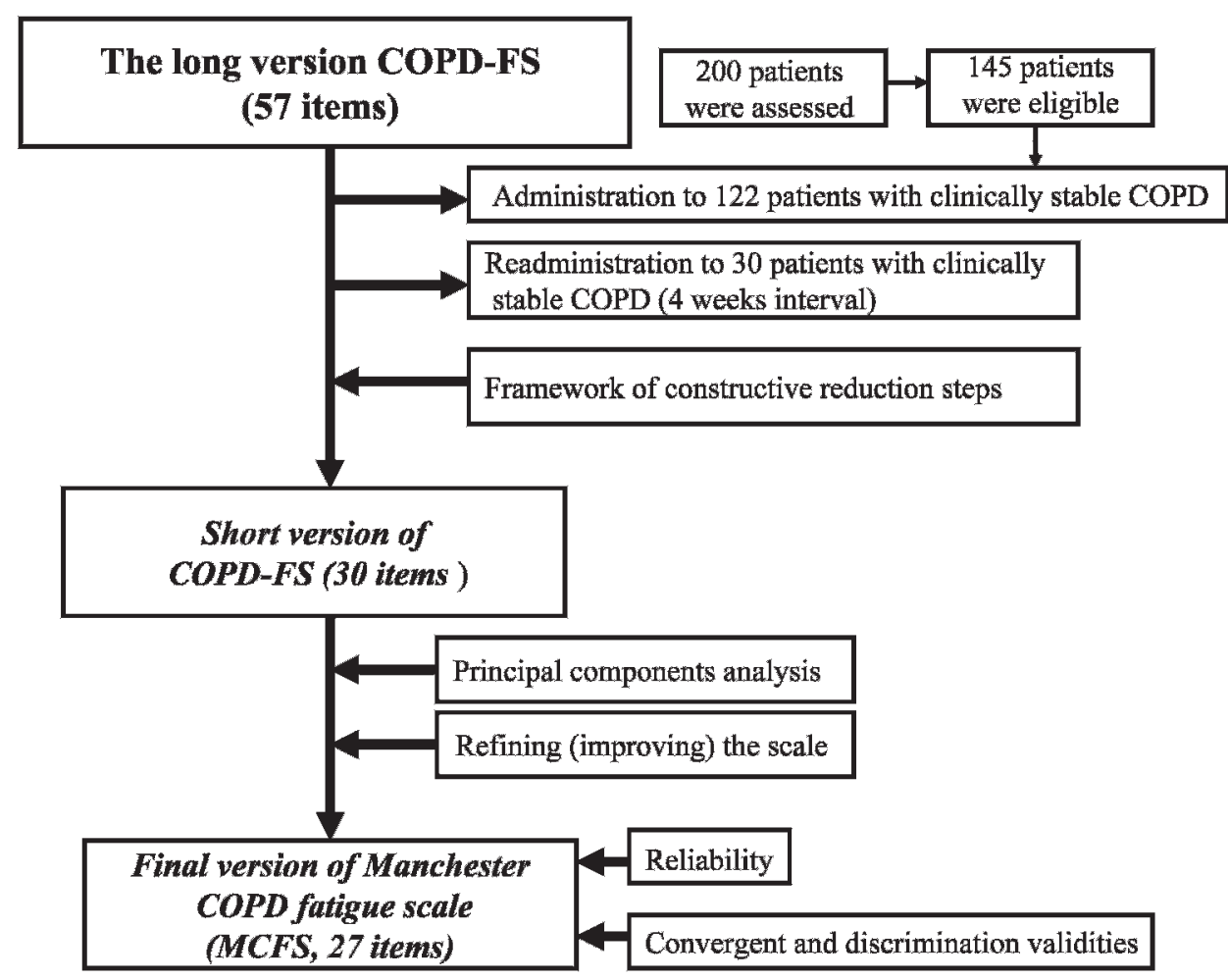

Obstructive Lung Disease) guidelines'; all patients had to be clinically stable for at least 4 weeks prior to the visit. Patients were not considered suitable if they had current or recurrent symptomatic ischaemic heart disease, lung cancer, known psychiatric illness, maintenance treatment with systemic corticosteroids, active tuberculosis or insulin-dependent diabetes mellitus. Patient recruitment extended from April 2006 to December 2007. We assessed 200 patients and from 145 suitable patients 122 were enrolled in the study.

All participants gave written informed consent. The Local Research Ethics Committee in Manchester approved the study.

\section{Measurements}

Spirometry was done according to the ATS/ERS Standardisation Guideline ${ }^{10}$ using a Jaeger MasterScreen spirometer (Jaeger, Hoechberg, Germany). Earlobe capillary blood gases were measured according to the method described by Spiro and Dowdeswell ${ }^{11}$ using a Radiometer analyser (Radiometer Medical, Copenhagen, Denmark). Exercise capacity was measured using the 6 minute walk distance (6MWD) test according to the ATS guideline. ${ }^{12}$ Before and after completion of the 6MWD test, dyspnoea and fatigue were assessed using the Borg scale. ${ }^{13}$

Health status was measured using the St George's Respiratory Questionnaire (SGRQ) ${ }^{14}$ and dyspnoea was assessed using the Medical Research Council (MRC) dyspnoea scale ${ }^{15}$; the multidimensional BODE index was also used. ${ }^{16}$ The Center for Epidemiological Study on Depression (CES-D) scale was used to assess depressive symptoms. ${ }^{17}$ Bioelectrical impedance analysis (BIA) was performed in the supine position at the beginning of the visit using Bodystat 1500 (Bodystat, Douglas, UK). The Functional Assessment of Chronic Illness Therapy (FACIT) fatigue scale of 13 items was also used in the 30 patients repeating our fatigue scale; overall scores of the FACIT scale range from 0 to 52 , with higher scores signifying less fatigue $^{7}$; this scale was not developed for patients with respiratory diseases but has previously been used in COPD. ${ }^{5}$

\section{Item reduction of fatigue scale}

The items reduction plan is shown in fig 1 and uses a constructive framework based on previous experiences. ${ }^{16}$ 18-20 Removed items are: (1) not applicable items - that is, items which patients reported were not applicable to their current lifestyle; (2) items showing redundancy of measurement defined by a high correlation $(r \geqslant 0.8)$ with another item; (3) items with a correlation coefficient $\left(r^{2}\right.$ coefficient $)<0.5$ or $>0.9$ with all the other items; ( $r^{2}$ coefficient is the value of the squared correlation between each item and the total score of all the other items); and (4) items with a "floor" or "ceiling" effect-that is, items that were mostly answered with "Never" or "Always". Importantly, before any item was removed, its clinical importance (content validity) was considered.

\section{Components extraction}

After the first item selection, the 30 items of this shortened COPD fatigue scale were subjected to an exploratory principal component analysis (PCA). We used Kaiser's criterion of eigenvalue $>1$ and Catell's scree test to determine the number of components. ${ }^{21}$

We conducted both orthogonal and oblique rotation techniques and found that oblique (Direct Oblimin) provided the best and easiest interpretation; nevertheless, the orthogonal approach (Varimax) provided a very similar result. To achieve a more consistent version, the shortened scale was resubjected twice to the PCA until no item loaded $<0.4$ on the components.

\section{Reliability of the fatigue scale}

To investigate the reliability of the shortened final fatigue scale we measured the test-retest intraclass correlation coefficient (ICC) of the scale and of each of its dimensions. Additionally, 
we compared the mean difference between two different measurements as described by Bland and Altman. ${ }^{22}$ We also investigated the internal consistency of the scale and each dimension. Cronbach's alpha coefficient ${ }^{23}$ was used and a correlation $\geqslant 0.7$ was assumed to indicate that questions within a dimension are likely to measure the same construct.

\section{Validity of the fatigue scale}

To examine the convergent validity, total and dimensional scores of the shortened final scale were correlated with the scores of the FACIT fatigue questionnaire and Borg scale before and after the 6MWD test. We also measured the correlation between total and dimensional scores of the fatigue scale and COPD severity, impairment in quality of life and the perception of dyspnoea using BODE scores, SGRQ and the MRC dyspnoea scale, respectively. The discriminant validity was also investigated using the mean fatigue scores between the original BODE quartiles, ${ }^{16}$ between depressed/not depressed patients (using $\geqslant 16$ scores cut-off of CES-D), and between patients with a $6 \mathrm{MWD}$ of more or less than $350 \mathrm{~m}^{24}$

\section{Method of administration and scoring system of the Manchester COPD fatigue scale (MCFS)}

Consistent with the original scale, the MCFS is a selfadministered scale investigating fatigue in the last 2 weeks, and the scoring system was stated as responses: Never, Rarely, Sometimes, Usually or Always. This was scored as 0, 0.5, 1, 1.5 or 2 , and thus the total score from the 27 items ranged from 0 to 54; the higher the score, the more fatigue.

\section{Statistical method}

Normal distribution was assessed by Kolmogorov-Smirnov goodness of fit test. In addition to the statistical methods mentioned previously, the Spearman correlation coefficient was used to examine the correlation between the fatigue scale and its dimensions with the Borg scale (before and after the 6MWD test), BODE scores and MRC dyspnoea scores. Paired t test was used to compare the mean of fatigue scores in both administrations.

The association of total and dimensional fatigue scores with COPD severity (using the original BODE quartiles) was examined using analysis of variance (ANOVA). We used the independent sample $t$ test to compare the mean fatigue score in groups defined according to CES-D depression scores and 6MWD. SPSS version 15 (SPSS, Chicago, Illinois, USA) was used.

\section{RESULTS}

The patient group consisted of 122 patients with mainly moderate stable COPD (mean forced expiratory volume in $1 \mathrm{~s}$ $\left(\mathrm{FEV}_{1}\right) 52 \%$ of predicted). The mean age was 66 years, and women made up $38 \%$ of the sample. As can be seen in table 1 , patients $<65$ years had more severe COPD, worse health status, higher depression scores, more dyspnoea, less muscle mass and were more likely to be smokers.

\section{Item reduction}

Using constructive steps, 27 items were excluded from the initial 57 items; these items are listed in table 1 in the online supplement. First, $>20 \%$ of the patients reported that items 21 , 35 and 45 were not applicable; second, a high correlation coefficient $(r \geqslant 0.8)$ between any two items was assessed, and items 5, 9, 13, 21, 31, 33, 36, 39, 47 and 51 were removed; third, each of items $1,2,3,6,12,15,17,18,19,20,21,26,34,35,38,44$,
45 and 55 showed a low $r^{2}$ coefficient; and fourth, items 6 and 45 showed clear floor and ceiling effects. After this procedure, we were left with a 30-item questionnaire.

\section{Dimensions}

The 30-item COPD fatigue scale was subjected to a PCA. Prior to performing the PCA, suitability of the data for factor analysis was assessed. Inspection of the correlation matrix revealed the presence of many coefficients of $\geqslant 0.3$. The Kaiser-Meyer-Oklin value was 0.94 (exceeding the recommended value of 0.6 ) and Bartlett's test of sphericity reached statistical significance $(<0.001)$, supporting the suitability of the data for a PCA.

PCA revealed the presence of three components with eigenvalues exceeding 1, explaining 57.1, 6.1 and $5.1 \%$ of the variance, respectively (table 2 in the online supplement). Using a scree test, it was decided to retain three components for further investigation (fig 1 in the online supplement). To aid in the interpretation of these three components, oblimin rotation was performed. The rotated solution revealed the presence of a simple structure, with all three components showing a significant number of strong loadings. Items related to physical activities were loaded to component 1 (physical component), items related to cognitive activities were loaded to component 2 (cognitive component) and items of psychosocial importance were loaded to component 3 (psychosocial component). Using the regression scores of each dimension there was a high correlation between the three components (table 5 in the online supplement), supporting the strong structure of the questionnaire. A summary of this step can be seen in table 2 in the online supplement.

In the final refining step, items with low loading (30, 50 and 54 according to their number in the original long version) were removed (as shown in table 2 in the online supplement). Removal of these items did not affect the total explained variance of the scale and provided better consistency and homogeneity for each component and within components. This step resulted in the 27-item final version of the MCFS with three dimensions: physical (11 items), cognitive ( 7 items) and psychosocial (9 items). The summary of pattern and structure matrices for PCA with oblimin rotation of the three-factor solution of the scale can be seen in table 3 in the online supplement.

The correlations between total score and each dimension were $>0.78$, and within dimensions were $>0.55$ ( $p<0.001$ for all correlations); further details can be seen in table 5 in the online supplement.

We found a high correlation between the total scores of the 27 -item final version of the questionnaire and those of the original 57-item long version $(r=0.99, p<0.001)$.

\section{Reliability of the MCFS}

For test-retest reliability the ICCs for the scale and its physical, cognitive and psychosocial dimensions were $0.97,0.96,0.91$ and 0.95 , respectively. The mean scores (SD) for total MCFS and the three dimensions at both visits are shown in table 2 .

A Bland-Altman plot showed small and statistically insignificant mean differences ( $p=0.8,0.5,0.6$ and 0.6 , respectively), and $95 \%$ limits of agreement of repeatability of -7 to +7.44 , -2.98 to $+3.42,-2.77$ to +3.07 and -3.25 to +3.55 , respectively; see figure 2 in the online supplement. Using Cronbach's alpha for measuring internal consistency for the scale and each dimension, we found values of $0.97,0.94,0.92$ and 0.95, respectively. 
Table 1 Baseline characteristics of the sample

\begin{tabular}{lllll}
\hline & All & $\geqslant \mathbf{6 5}$ years old & $<\mathbf{6 5}$ years old & p Value \\
\hline Number & 122 & $77(63 \%)$ & $45(37 \%)$ & \\
$\%$ Men & $62 \%$ & $66 \%$ & $53 \%$ & 0.2 \\
$\%$ Current smokers & $29 \%$ & $25 \%$ & $36 \%$ & 0.28 \\
Pack/years, median (IOR) & $40(25.8)$ & $40.7(24.9)$ & $36(25)$ & 0.21 \\
$\mathrm{FEV} \%$ & $52.5(18.5)$ & $53.6(16)$ & $49(21)$ & 0.2 \\
$\mathrm{PaO}_{2}(\mathrm{kPa})$ & $9.2(1.4)$ & $9.1(1)$ & $9.2(1.3)$ & 0.7 \\
$\mathrm{PaCO}_{2}(\mathrm{kPa})$ & $5.2(0.58)$ & $5.1(0.5)$ & $5.3(0.7)$ & 0.08 \\
$\mathrm{BMI}\left(\mathrm{kg} / \mathrm{m}^{2}\right)$ & $27.5(5.8)$ & $27.8(5.2)$ & $27.1(6.7)$ & 0.6 \\
$\mathrm{FFMI}\left(\mathrm{kg} / \mathrm{m}^{2}\right)$ & $17.9(3.3)$ & $18.1(3.1)$ & $17.7(3.8)$ & 0.5 \\
$\mathrm{MRC}$ median (IOR) & $1(1)$ & $1(1)$ & $2(2)$ & 0.059 \\
$\mathrm{CES}-\mathrm{D}$ median (IOR) & $10(12)$ & $8(10)$ & $12(12)$ & 0.006 \\
$\mathrm{SGRO}$ total score & $46(20)$ & $42(17.8)$ & $54(21.9)$ & 0.002 \\
Symptoms domain & $55.4(23.1)$ & $52(21)$ & $61(25)$ & 0.04 \\
Activity domain & $62.4(27)$ & $56.7(25)$ & $72(27)$ & 0.003 \\
Impact domain & $35.1(19.7)$ & $31(17)$ & $42.3(22)$ & 0.004 \\
\hline
\end{tabular}

Mean values and SDs are shown unless otherwise noted.

BMI, body mass index; CES-D, Center for Epidemiologic Studies Depression scale; FEV $\%$, forced expiratory volume over $1 \mathrm{~s}$ of predicted; FFMI, fat-free mass index; IQR, interquartile range; MRC, Medical Research Council dyspnoea $\mathrm{Scale}$; $\mathrm{PaCO}_{2}$, arterial carbon dioxide pressure; $\mathrm{PaO}_{2}$, arterial oxygen partial pressure; SGRO, St George's Respiratory Questionnaire.

\section{Validity of the MCFS}

Regarding convergent validity, the scale showed a high correlation with the FACIT fatigue scale and fatigue in the Borg scale. It also responded well to the change in fatigue measured with the Borg scale before and after the 6MWD test, as shown in table 3 .

Additionally, the MCFS showed superiority to the FACIT scale in correlation with these well-established measures in COPD as shown in table 4 in the online supplement.

Using the original BODE quartiles, we found increasing fatigue with increasing disease severity; as can be seen in table 4, the mean total and dimensional fatigue scores were significantly higher consistently with the degree of COPD severity.

We found that $48 \%$ of our patients suffered from limited exercise capacity defined as a $6 \mathrm{MWD}<350 \mathrm{~m}$. Our fatigue scale showed significant discriminating ability of poor exercise performance. In addition, patients with depressive symptoms scored approximately twice as high on total fatigue and dimensions, as shown in table 4 . The scale also correlated well with health status and dyspnoea as shown in table 3 . Additionally, the MCFS scale showed superiority to the FACIT scale in correlation with these well-established measures in COPD as shown in table 4 in the online supplement.

\section{Fatigue, gender and age}

We found no gender difference in fatigue scores. However, age had a weak negative correlation with fatigue scores; younger patients ( $<65$ years) perceived more fatigue than older patients,

Table 2 Comparison of mean (SD) scores of the total MCFS and its dimensions at both visits (30 day interval)

\begin{tabular}{lccll}
\hline & $\begin{array}{l}\text { Mean score } \\
\text { (SD) first } \\
\text { completion }\end{array}$ & $\begin{array}{l}\text { Mean score } \\
\text { (SD) second } \\
\text { completion }\end{array}$ & $\begin{array}{l}\text { Mean } \\
\text { difference (SD) }\end{array}$ & p Value \\
\hline $\begin{array}{l}\text { Total MCFS score } \\
\text { Physical }\end{array}$ & $23(14)$ & $23.2(13.4)$ & $-0.22(3.6)$ & 0.8 \\
dimension & $9.2(5.9)$ & $9.4(5.6)$ & $-0.22(1.6)$ & 0.47 \\
$\begin{array}{l}\text { Cognitive } \\
\text { dimension }\end{array}$ & $5.4(3.5)$ & $5.5(3.2)$ & $-0.15(1.5)$ & 0.58 \\
$\begin{array}{l}\text { Psychosocial } \\
\text { dimension }\end{array}$ & $8.4(5.2)$ & $8.3(5.2)$ & $0.15(1.7)$ & 0.6 \\
\hline
\end{tabular}

MCFS, Manchester chronic obstructive pulmonary disease fatigue scale.
31.9 vs $24.4(p=0.004)$ for total fatigue and 12.3 vs 9.7 $(p=0.02), 7.6$ vs $5.7(p=0.007)$ and 12.0 vs $9.0(p=0.003)$ for physical, cognitive and psychosocial fatigue, respectively.

\section{DISCUSSION}

This study presents a novel reliable, valid and simple scale for measuring fatigue in patients with COPD, the MCFS. We found that its 27 items loaded in a balanced distribution on three dimensions: physical, cognitive and psychosocial fatigue. The total scale and each dimension had optimal internal consistency and showed a significant correlation with other available scales measuring fatigue such as the FACIT fatigue and Borg scales. The scale had meaningful discriminating ability in identifying patients with poor exercise performance, low mood and severe disease according to BODE score. The scale also achieved a significant correlation with other established valid constitutional measures in COPD such as the SGRQ and MRC dyspnoea scale.

We started out with a pool of 57 items in an early draft of the COPD-specific fatigue scale. However, this draft appeared to have redundant items and was time consuming. In our study, patients did not seem to have a problem with responding to questions on the fatigue scale and we actually found that $>94 \%$ of the patients completed the long fatigue questionnaire at both visits without omitting items. We found that the completeness of the fatigue scale was better than both the CES-D and SGRO questionnaires. This of course needs validation in other studies as previous studies have in fact shown some issues with data completeness with the CES-D and SGRQ questionnaires ${ }^{25} 26$ and the same could be the case for the MCFS outside the research setting. The high correlation between the total scores of the 27item short version and the early 57 -item long version makes us confident that our reduction steps were reliable and valid. Readministration of the scale after a 1-month interval to stable patients was considered important in light of the chronicity of COPD. ${ }^{27}$ The scale was administered to a gender-balanced sample in both administration sessions. Thus, the choice of items is unlikely to be affected by gender. Consistent with the literature, we found no correlation between gender and fatigue. ${ }^{5}$ The negative correlation between age and fatigue follows the pattern seen for MRC dyspnoea, SGRQ and CES-D scores in table 1. 
Table 3 Correlations between total and dimensional scores of the MCFS and the FACIT fatigue scale, Borg scale, BODE index, SGRO and MRC dyspnoea scale

\begin{tabular}{|c|c|c|c|c|c|c|c|c|c|}
\hline & $\begin{array}{l}\text { FACIT fatigue } \\
\text { scale }\end{array}$ & $\begin{array}{l}\text { Fatigue (Borg } \\
\text { scale) at } \\
\text { baseline }\end{array}$ & $\begin{array}{l}\text { Fatigue (Borg } \\
\text { scale) after } \\
\text { 6MWD }\end{array}$ & BODE scores & Total SGRO & Symptom & Activity & Impact & $\begin{array}{l}\text { MRC dyspnoea } \\
\text { scale }\end{array}$ \\
\hline Total fatigue score & -0.81 & 0.53 & 0.63 & 0.46 & 0.8 & 0.51 & 0.72 & 0.79 & 0.51 \\
\hline Cognitive dimension & -0.78 & 0.5 & 0.5 & 0.29 & 0.62 & 0.39 & 0.56 & 0.61 & 0.38 \\
\hline $\begin{array}{l}\text { Psychosocial } \\
\text { dimension }\end{array}$ & -0.84 & 0.48 & 0.56 & 0.42 & 0.74 & 0.47 & 0.65 & 0.76 & 0.45 \\
\hline
\end{tabular}

All presented correlations have a $p$ value $<0.001$

BODE, multidimensional index ( $B$, body mass index; 0 , obstruction of airways as measured by forced expiratory volume in $1 \mathrm{~s}$; $D$, dyspnoea as measured by the MRC scale; E, exercise capacity as measured by the 6MWD test); FACIT, Functional Assessment of Chronic Illness Therapy; MCFS, Manchester chronic obstructive pulmonary disease fatigue scale; MRC, Medical Research Council; 6MWD, 6 minute walk distance; SGRO, St George's Respiratory Questionnaire.

Table 4 MCFS scores in groups defined according to BODE quartiles, CES-D scores and 6MWD

\begin{tabular}{|c|c|c|c|c|c|c|c|c|}
\hline & \multicolumn{4}{|c|}{ BODE quartiles (0s) } & \multicolumn{2}{|l|}{ Depression } & \multicolumn{2}{|l|}{ 6MWD } \\
\hline & 01 & 02 & 03 & 04 & Not depressed & Depressed & $6 M W D \geqslant 350 \mathrm{~m}$ & 6MWD $<350 \mathrm{~m}$ \\
\hline Total fatigue score & $20.8(12.2)$ & $24.7(10.2)$ & $34.9(9.7)$ & $39.4(10.5)$ & $22.9(12)$ & $40.9(9.5)$ & $18.6(11.9)$ & $31.5(9.8)$ \\
\hline Cognitive dimension & $5(3.3)$ & $5.5(2.8)$ & $7.2(2.9)$ & $8(3.9)$ & $5.4(3.3)$ & $9.7(3.1)$ & $4.2(3.1)$ & $7.1(2.8)$ \\
\hline Psychosocial dimension & $7.8(4.9)$ & $8.8(4.2)$ & $13.5(4.2)$ & $15(4.1)$ & $8.4(4.8)$ & $15.5(4.1)$ & $7.1(4.9)$ & $11.6(4.2)$ \\
\hline
\end{tabular}

Mean values and SDs are shown.

All presented values have a $p$ value $<0.001$ except for the difference of cognitive fatigue scores between BODE quartiles where $p=0.015$

BODE, multidimensional index (B, body mass index; 0 , obstruction of airways as measured by forced expiratory volume in $1 \mathrm{~s}$; $\mathrm{D}$, dyspnoea as measured by the Medical Research Council scale; E, exercise capacity as measured by the 6MWD test); CES-D, Center for Epidemiologic Studies Depression scale; MCFS, Manchester chronic obstructive pulmonary disease fatigue scale; 6MWD, 6 minute walk distance.

Fatigue has been studied as a component in previous scales examining health status. As an example, the CRO has a fatigue component but this is assessed crudely by use of a few general questions. ${ }^{1}$ Other general scales have been used in a limited number of studies, including the FACIT fatigue scale, ${ }^{7}$ the Piper Fatigue Scale (PFS), ${ }^{28}$ the Multidimensional Fatigue Index 20 $(\mathrm{MFI}-20)^{29}$ and the Fatigue Impact Scale (FIS). ${ }^{30}$ None of these is COPD specific. The FACIT scale has 13 items and it only offers a simple assessment, the PFS is a 41-point visual analogue scale and it was found to be difficult to follow, and the MFI-20 assesses fatigue for the last few days only. ${ }^{2}$ The FIS of 40 items offered 20 items to assess psychosocial aspects but only 10 items to describe fatigue in response to physical activities.

A recent study using the FACIT fatigue scale found that overall fatigue correlated with poor quality of life, dyspnoea, depression and annual exacerbation frequency. ${ }^{5}$ In our study we found that cognitive and psychosocial fatigue dimensions also correlated significantly with COPD severity, impairment of quality of life, poor exercise performance and low mood, and our findings underscore the need to examine psychosocial and cognitive fatigue aspects in detail in COPD. Another feature of this extrapulmonary domain, depression, has in different studies been associated with poor exercise performance, ${ }^{31}$ exacerbation, $^{32}$ hospital admission ${ }^{33}$ and mortality. ${ }^{34}$

Our study has weaknesses. We cannot preclude that part of the fatigue measured may relate to factors other than COPD not captured sufficiently-for example, other undiagnosed chronic diseases. However, this is a feature of all measures of overall health outcomes and we made an effort to question patients on co-morbidities. Fatigue could possibly be affected for long after an exacerbation, in line with findings regarding health status $^{35}$; we chose 4 weeks of documented stability as this is generally regarded as a valid time interval.

In conclusion, the MCFS has shown significant reliability, reproducibility and validity, and measured dimensions of fatigue in moderate stable COPD. It is short, simple to complete, easy to administer/self-administer and investigators do not need training for administration or scoring the scale. In addition to overall fatigue assessment, the scale offers dimensional measurements. We recommend further studies of fatigue in COPD and propose to include the MCFS as a tool (a copy of the MCFS is available in the online supplement).

Acknowledgements: We would like to thank Professor Ingela Wiklund for the inspiring suggestions, and our institution's medical statistician Dr Julie Morris for statistical advice.

Competing interests: None.

Ethics approval: The Local Research Ethics Committee in Manchester approved the study.

Provenance and peer review: Not commissioned; externally peer reviewed.

\section{REFERENCES}

1. Guyatt GH, Berman LB, Townsend M, et al. A measure of quality of life for clinical trials in chronic lung disease. Thorax 1987;42:773-8.

2. Breslin $\mathbf{E}$, van der Schans $C$, Breukink $S$, et al. Perception of fatigue and quality of life in patients with COPD. Chest 1998;114:958-64.

3. Theander $\mathbf{K}$, Unosson M. Fatigue in patients with chronic obstructive pulmonary disease. J Adv Nurs 2004;45:172-7.

4. Kapella MC, Larson JL, Patel MK, et al. Subjective fatigue, influencing variables, and consequences in chronic obstructive pulmonary disease. Nurs Res 2006;55:10-7.

5. Baghai-Ravary R, Quint JK, Goldring JJ, et al. Determinants and impact of fatigue in patients with chronic obstructive pulmonary disease. Respir Med 2009;103:216-23.

6. Kinsman RA, Fernandez E, Schocket M, et al. Multidimensional analysis of the symptoms of chronic bronchitis and emphysema. J Behav Med 1983;6:339-57.

7. Yellen SB, Cella DF, Webster K, et al. Measuring fatigue and other anemia-related symptoms with the Functional Assessment of Cancer Therapy (FACT) measurement system. J Pain Symptom Manage 1997;13:63-74.

8. Small S, Lamb M. Fatigue in chronic illness: the experience of individuals with chronic obstructive pulmonary disease and with asthma. J Adv Nurs 1999;30:469-78.

9. GOLD Scientific Committee. Global strategy for the diagnosis, management and prevention of chronic obstructive pulmonary disease. 2004. www goldcopd org (accessed 12Jan 2006)

10. Miller MR, Crapo R, Hankinson J, et al. Standardisation of spirometry. Eur Resp J 2005:26:319-38. 
11. Spiro SG, Dowdeswell IRG. Arterialized ear lobe blood samples for blood gas tensions. Br J Dis Chest 1976;70:263-8.

12. American Thoracic Society. ATS Statement: guidelines for the six-minute walk test. Am J Respir Crit Care Med 2002;166:111-7.

13. Borg GA. Psychophysical bases of perceived exertion. Med Sci Sports Exerc 1982;14:377-81.

14. Jones PW, Quirk FH, Baveystock CM, et al. A self-complete measure of health status for chronic airflow limitation. The St. George's Respiratory Questionnaire. Am Rev Respir Dis 1992;145:1321-7.

15. Bestall JC, Paul EA, Garrod R, et al. Usefulness of the Medical Research Council (MRC) dyspnoea scale as a measure of disability in patients with chronic obstructive pulmonary disease. Thorax 1999;54:581-6.

16. Celli BR, Cote CG, Marin JM, et al. The body-mass index, airflow obstruction, dyspnea, and exercise capacity index in chronic obstructive pulmonary disease. N Engl J Med 2004;350:1005-12.

17. Radloff LS. The CES-D scale: a self-report depression scale for research in the general population. Appl Psychol Measur 1977;1:385-401.

18. Wiklund IK, Fullerton S, Hawkey CJ, et al. An irritable bowel syndrome-specific symptom questionnaire: development and validation. Scand J Gastroenterol 2003;38:947-54.

19. O'Leary CJ, Jones PW. The influence of decisions made by developers on health status questionnaire content. Qual Life Res 1998;7:545-50.

20. O'Leary CJ, Jones PW. The left ventricular dysfunction questionnaire (LVD-36): reliability, validity, and responsiveness. Heart 2000;83:634-40.

21. Tabachnick B, Fidell L. Using multivariate statistics. Boston: Allyn and Bacon, 2007:607-75.

22. Bland JM, Altman DG. Statistical methods for assessing agreement between two methods of clinical measurement. Lancet 1986:1:307-10.

23. Cronbach LJ. Coefficient alpha and the internal structure of tests. Psychometrica 1951;16:297-334.
24. Cote CG, Casanova C, Marin JM, et al. Validation and comparison of reference equations for the 6-min walk distance test. Eur Respir J 2008;31:571-8.

25. van Manen JG, Bindels PJ, Dekker FW, et al. Risk of depression in patients with chronic obstructive pulmonary disease and its determinants. Thorax 2002:57:412-6.

26. Ferrer M, Alonso J, Prieto L, et al. Validity and reliability of the St George's Respiratory Questionnaire after adaptation to a different language and culture: the Spanish example. Eur Respir J 1996;9:1160-6.

27. Yohannes AM, Roomi J, Winn S, et al. The Manchester Respiratory Activities of Daily Living questionnaire: development, reliability, validity, and responsiveness to pulmonary rehabilitation. J Am Geriatr Soc 2000;48:1496-500.

28. Piper B, Lindsey A, Dodd M, et al. The development of an instrument to measure the subjective dimension of fatigue. In: Funk SGG, Tornquist EM, Champagne MT, et al.Key aspects of comfort: management of pain, fatigue and nausea. New York: Springer-Verlag, 1989:199-208.

29. Smets EM, Garssen B, Bonke B, et al. The Multidimensional Fatigue Inventory (MFI) psychometric qualities of an instrument to assess fatigue. J Psychosom Res 1995; 39:315-25.

30. Fisk JD, Ritvo PG, Ross $L$, et al. Measuring the functional impact of fatigue: initial validation of the fatigue impact scale. Clin Infect Dis 1994;18(Suppl 1):S79-83.

31. Al-shair K, Dockry R, Mallia-Milanes B, et al. Depression and its relationship with poor exercise capacity, BODE index and muscle wasting in COPD. Respir Med (doi:10.1016/j.rmed.2008.11.021)

32. Quint JK, Baghai-Ravary R, Donaldson GC, et al. Relationship between depression and exacerbations in COPD. Eur Respir J 2008;32:53-60.

33. Dahlen I, Janson C. Anxiety and depression are related to the outcome of emergency treatment in patients with obstructive pulmonary disease. Chest 2002; 122:1633-7.

34. Almagro $\mathbf{P}$, Calbo E, Ochoa de Echaguen $\mathrm{A}$, et al. Mortality after hospitalization for COPD. Chest 2002:121:1441-8.

35. Spencer S, Jones PW, GLOBE Study Group. Time course of recovery of health status following an infective exacerbation of chronic bronchitis. Thorax 2003;58:589-93.

\section{Quality \& Safety in Health Care}

Quality \& Safety in Health Care is a leading international peer-review journal in the growing area of quality and safety improvement. It provides essential information for those wanting to reduce harm and improve patient safety and the quality of care. The journal reports and reflects research, improvement initiatives and viewpoints and other discursive papers relevant to these crucial aims with contributions from researchers, clinical professionals and managers and experts in organisational development and behaviour.

qshc.bmj.com

Quality

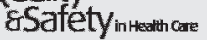

